\title{
Equivalence of Uniqueness in Law and Joint Uniqueness in Law for SDEs Driven by Poisson Processes
}

\author{
Huiyan Zhao', Chunhua $\mathrm{Hu}^{1}$, Siyan $\mathrm{Xu}^{2}$ \\ ${ }^{1}$ School of Applied Mathematics, Beijing Normal University Zhuhai, Zhuhai, China \\ ${ }^{2}$ School of Science, Ningbo University, Ningbo, China \\ Email: huiyan.zhao@msn.com, hchw006@aliyun.com, xusiyan@nbu.edu.cn
}

Received 23 November 2015; accepted 17 May 2016; published 20 May 2016

Copyright (C) 2016 by authors and Scientific Research Publishing Inc.

This work is licensed under the Creative Commons Attribution International License (CC BY).

http://creativecommons.org/licenses/by/4.0/

(c) () Open Access

\begin{abstract}
We give an extension result of Watanabe's characterization for 2-dimensional Poisson processes. By using this result, the equivalence of uniqueness in law and joint uniqueness in law is proved for one-dimensional stochastic differential equations driven by Poisson processes. After that, we give a simplified Engelbert theorem for the stochastic differential equations of this type.
\end{abstract}

Keywords

Uniqueness in Law, Joint Uniqueness in Law, Poisson Process, Engelbert Theorem

\section{Introduction}

There are several types of solutions and uniqueness for stochastic differential equations, such as strong solution, weak solution, pathwise uniqueness, uniqueness in law and joint uniqueness in law, which will be introduced in Section 2. The relationship between them was firstly studied by Yamada and Watanabe [1]. They got

$$
\text { Pathwise uniqueness } \Rightarrow \text { Uniqueness in law }
$$

and

$$
\text { Weak solution + Pathwise uniqueness } \Rightarrow \text { Strong solution, }
$$

which is the famous Yamada-Watanabe theorem. It's an important method to prove the existence of strong solution for SDEs Nowadays. The study on this topic is still alive today and new papers are published, see [2]-[10]. On the other hand, Jacod [11] and Engelbert [12] extended the Yamada-Watanabe theorem to the 
stochastic differential equation driven by semi-martingales. Especially, Engelbert got an inverse result, that is

$$
\text { Strong solution + Joint uniqueness in law } \Rightarrow \text { Pathwise uniqueness, }
$$

which can be seen as a complement of the Yamada-Watanabe theorem. Recently, Kurtz [5] [7] considered an abstract stochastic equation of the form

$$
\Gamma(X, Y)=0,
$$

where $\Gamma: S_{1} \times S_{2} \rightarrow \mathbb{R}, \quad S_{1}$ and $S_{2}$ are Polish spaces. They obtained an unified result ([7] Theorem 1.5):

$$
\begin{array}{|l|}
\hline \text { Strong solution + Joint uniqueness in law } \\
\mathbb{1} \\
\text { Weak solution + Pathwise uniqueness }
\end{array}
$$

which was called the Yamada-Watanabe-Engelbert thereom. This result can cover most results mentioned above. However, joint uniqueness in law is harder to check than uniqueness in law in view of application. The natural question that arises now is: under what conditions, joint uniqueness can be equivalent to uniqueness in law? Kurtz ([5] [7]) gave a positive answer for the stochastic equations of the form

$$
f_{i}(X, Y)=0 \text {, a.s., } i \in I \text {, }
$$

when the constrains are simple (linear) equations. It's sad that the stochastic differential equations are not of the form above, therefore the equivalence does not follow from this result.

There exist few results for this question. As far as we know, Cherny [14] and Brossard [13] proved the equivalence of uniqueness in law and joint uniqueness in law for Itô equations of the following type

$$
X_{t}=x+\int_{0}^{t} b(t, X) \mathrm{d} t+\int_{0}^{t} \sigma(t, X) \mathrm{d} B_{t}
$$

driven by Brownian motion with the coefficients which only need to be measurable. Later, Qiao [15] extended the result of [14] to a type of infinite dimensional stochastic differential equaion. For stochastic differential equations with jumps, there is still no such result. So, in this paper, we are concerned with the following onedimensional stochastic differential equation driven by Poisson process

$$
X_{t}=x+\int_{0}^{t} b(s, X) \mathrm{d} t+\int_{0}^{t} g(s, X) \mathrm{d} N_{s} .
$$

We will give an extension form of Watanabe's characterization for 2-dimensional Poisson process, then by applying Cherny's approach, we prove the equivalence of the uniqueness in law and joint uniqueness in law for Equation (1).

This paper is organized as follows. In Section 2, we prepapre some notations and some definitions. After that, the main results are given and proved in Section 3.

\section{Notations and Definitions}

Let $\mathbb{D}:=D\left(\mathbb{R}_{+}, \mathbb{R}\right)$ be the space of all càdlàg functions: $\mathbb{R}_{+} \rightarrow \mathbb{R}$ and let $\mathcal{B}_{t}(\mathbb{D})$ denote the $\sigma$-algebra generated by all the maps $\pi_{s}: \mathbb{D} \rightarrow \mathbb{R}, \quad s \geq 0$, where $\pi_{s}(\omega):=\omega_{s}, \omega \in \mathbb{D}$. Let $\mathcal{B}(\mathbb{D}):=\vee_{t \geq 0} \mathcal{B}_{t}(\mathbb{D})$.

Definition 2.1. Let $(\Omega, \mathcal{F}, P)$ be a probability space with a given filtration $\mathbf{F}=\left(\mathcal{F}_{t}\right)_{t \geq 0}$, and let $t \rightarrow \lambda_{t}$ be a deterministic function of time. A counting process $N$ is a Poisson process with intensity function $\lambda$ with respect to the filtration $\mathbf{F}$ if it satisfies the following conditions.

1) $N$ is adapted to $\mathbf{F}$;

2) For all $s \leq t$ the random variable $N_{t}-N_{s}$ is independent of $\mathcal{F}_{s}$;

3) For all $s \leq t$, the conditional distribution of the increment $N_{t}-N_{s}$ is given by

$$
P\left(N_{t}-N_{s}=k \mid \mathcal{F}_{s}\right)=\mathrm{e}^{-\Lambda_{s, t}} \frac{\left(\Lambda_{s, t}\right)^{k}}{k !}, \quad k=0,1,2, \cdots,
$$


where

$$
\Lambda_{s, t}=\int_{s}^{t} \lambda_{u} \mathrm{~d} u
$$

Definition 2.2. Let $(\Omega, \mathcal{F}, P)$ be a probability space with a given filtration $\mathbf{F}=\left(\mathcal{F}_{t}\right)_{t \geq 0}$, and let $t \rightarrow \lambda_{t}^{j}$, $j=1,2$ be two deterministic function of time. $N^{1}$ and $N^{2}$ are two $\boldsymbol{F}$-Poisson processes with intensity function $\lambda^{1}$ and $\lambda^{2}$ respectively. Process $\mathbf{N}:=\left(N^{1}, N^{2}\right)$ is called a 2-dimensional $\mathbf{F}$-Poisson process with intensity function $\left(\lambda^{1}, \lambda^{2}\right)$ if $N^{1}$ and $N^{2}$ are independent.

We have the following Watanabe characterization for one dimensional Poisson process (see [16]).

Lemma 2.3. Let $(\Omega, \mathcal{F}, P)$ be a probability space with a given filtration $\mathbf{F}=\left(\mathcal{F}_{t}\right)_{t \geq 0}$. Assume that $N$ is a counting process and that $t \rightarrow \lambda_{t}$ is a deterministic function. Assume furthermore that the process $M$, defined by

$$
M_{t}:=N_{t}-\int_{0}^{t} \lambda_{s} \mathrm{~d} s,
$$

is an $\mathbf{F}$-martingale. Then $N$ is a $\mathbf{F}$-Poisson process with intensity function $\lambda$.

In this paper, we consider the following stochastic differential equation driven by the Poisson process

$$
X_{t}=X_{0}+\int_{0}^{t} b(s, X) \mathrm{d} t+\int_{0}^{t} g(s, X) \mathrm{d} N_{s},
$$

where $b: \mathbb{R}_{+} \times \mathbb{D} \rightarrow \mathbb{R}$ and $g: \mathbb{R}_{+} \times \mathbb{D} \rightarrow \mathbb{R}$ are $\mathcal{B}\left(\mathbb{R}_{+}\right) \otimes \mathcal{B}(\mathbb{D}) / \mathcal{B}(\mathbb{R})$ - measurable and for each $t \in \mathbb{R}_{+}$, $x \in \mathbb{D}, b(t, x)$ and $g(t, x)$ are predictable.

Definition 2.4. A pair $(X, N)$, where $X=(X(t))_{t \in[0, \infty)}$ is a càdlàg $\left(\mathcal{F}_{t}\right)$-adapted process with paths in $\mathbb{D}$ and $N$ is a Poisson process with intensity function $\lambda$ on a stochastic basis $\left(\Omega, \mathcal{F}, P,\left(\mathcal{F}_{t}\right)\right)$, is called a weak solution of (2.1) if

1) For all $t \in[0, \infty)$,

$$
\int_{0}^{t}|b(s, X)| \mathrm{d} s+\int_{0}^{t}|g(s, X)| \mathrm{d} N_{s}<\infty \quad P \text {-a.e.; }
$$

2) For all $t \in[0, \infty)$,

$$
X(t)=x+\int_{0}^{t} b(s, X) \mathrm{d} s+\int_{0}^{t} g(s, X) \mathrm{d} N_{s} \quad P \text {-a.e.. }
$$

Definition 2.5. We say that uniqueness in law holds for (2.1) if whenever $(X, N)$ and $\left(X^{\prime}, N^{\prime}\right)$ are two weak solutions with stochatic bases $\left(\Omega, \mathcal{F}, P,\left(\mathcal{F}_{t}\right)\right)$ and $\left(\Omega^{\prime}, \mathcal{F}^{\prime}, P^{\prime},\left(\mathcal{F}_{t}^{\prime}\right)\right)$ such that

$$
\operatorname{Law}\left(X_{0}\right)=\operatorname{Law}\left(X_{0}^{\prime}\right) \text { on } \mathbb{R},
$$

then

$$
\operatorname{Law}(X)=\operatorname{Law}\left(X^{\prime}\right) \quad \text { on } \mathbb{D} \text {. }
$$

Definition 2.6. We say that joint uniqueness in law holds for (2.1) if whenever $(X, N)$ and $\left(X^{\prime}, N^{\prime}\right)$ are two weak solutions with stochatic bases $\left(\Omega, \mathcal{F}, P,\left(\mathcal{F}_{t}\right)\right)$ and $\left(\Omega^{\prime}, \mathcal{F}^{\prime}, P^{\prime},\left(\mathcal{F}_{t}^{\prime}\right)\right)$ such that

$$
\operatorname{Law}\left(X_{0}\right)=\operatorname{Law}\left(X_{0}^{\prime}\right) \text { on } \mathbb{R} \text {, }
$$

then

$$
\operatorname{Law}(X, N)=\operatorname{Law}\left(X^{\prime}, N^{\prime}\right) \quad \text { on } \mathbb{D} \times \mathbb{D} \text {. }
$$


Definition 2.7. We say that pathwise uniqueness holds for (1.1) if whenever $(X, N)$ and $\left(X^{\prime}, N\right)$ are two weak solutions on the same stochatic bases $\left(\Omega, \mathcal{F}, P,\left(\mathcal{F}_{t}\right)\right)$ such that $X(0)=X^{\prime}(0)$ P-a.s., then $P$-a.s.

$$
X(t)=X^{\prime}(t) \quad t \in[0, \infty)
$$

\section{Main Results}

Theorem 3.1. Suppose that the uniqueness in law holds for (2.1). Then, for any solutions $(X, N)$ and $(\tilde{X}, \tilde{N})$, the law of $(X, N)$ and the law of $(\tilde{X}, \tilde{N})$ are equal on $\mathbb{D} \times \mathbb{D}$, that is

$$
\operatorname{Law}(X, N)=\operatorname{Law}(\tilde{X}, \tilde{N}) .
$$

According to Theorem 1.5 of Kurtz [7], we have the following simplified Yamada-Watanabe-Engelbert theorem (see aslo [12] Theorem 3, [14] Theorem 3.2) immediately.

Corollary 3.2. The following are equivalent:

1) Equation (2.1) has a strong solution and uniqueness in law holds;

2) Equation (2.1) has a weak solution and pathwise uniqueness holds.

$$
\begin{aligned}
& \text { Strong solution + Uniqueness in law } \\
& \mathbb{1} \\
& \text { Weak solution + Pathwise uniqueness }
\end{aligned}
$$

We have the following generalised martingale characterization for 2-dimensional Poisson processes, which may have its own interest.

Lemma 3.3. Let $(\Omega, \mathcal{F}, P)$ be a probability space with a given filtration $\mathbf{F}=\left(\mathcal{F}_{t}\right)_{t \geq 0}$. Assume that $\mathbf{N}=\left(N^{1}, N^{2}\right)$ is a 2-dimensional counting process and that $t \rightarrow \lambda_{t}^{i}, i=1,2$ are two deterministic function. Then, $\mathbf{N}$ is a 2-dimensional $\mathbf{F}$-Poisson process with intensity function $\left(\lambda^{1}, \lambda^{2}\right)$ is equivalent to the following two conditions.

1) Processes $M^{1}$ and $M^{2}$ defined by

$$
M_{t}^{i}:=N_{t}^{i}-\int_{0}^{t} \lambda_{s}^{i} \mathrm{~d} s, \quad i=1,2,
$$

are $\mathbf{F}$-martingales.

2) Process $N$ defined by

$$
N_{t}:=N_{t}^{1}+N_{t}^{2}
$$

is a $\mathbf{F}$-Poisson process with intensity function $\lambda^{1}+\lambda^{2}$.

Proof. By Lemma 2.3, we only need to prove that two Poisson processes are independent if and only if their sum is also a Poisson process.

Suppose that $N^{1}$ and $N^{2}$ are two independent Poisson processes. For $N_{t}:=N_{t}^{1}+N_{t}^{2}$, we have,

$$
\begin{aligned}
& P\left(\bigcup_{t \geq 0}\left\{\Delta N_{t}^{1}=1, \Delta N_{t}^{2}=1\right\}\right) \\
& =P\left(\bigcup_{i=1}^{\infty}\left\{\Delta N_{t_{i}}^{1}=1, \Delta N_{t_{i}}^{2}=1\right\}\right) \\
& \leq \sum_{i=1}^{\infty} P\left(\left\{\Delta N_{t_{i}}^{1}=1, \Delta N_{t_{i}}^{2}=1\right\}\right) \\
& \leq \sum_{i=1}^{\infty} \lim _{h \rightarrow 0^{+}} P\left(\left\{N_{t_{i}}^{1}-N_{t_{i}-h}^{1}=1, N_{t_{i}}^{2}-N_{t_{i}-h}^{2}=1\right\}\right) .
\end{aligned}
$$


By the independence of $N^{1}$ and $N^{2}$, for each $t_{i}$, we obtain

$$
\begin{aligned}
& \lim _{h \rightarrow 0^{+}} P\left(\left\{N_{t_{i}}^{1}-N_{t_{i}-h}^{1}=1, N_{t_{i}}^{2}-N_{t_{i}-h}^{2}=1\right\}\right) \\
& =\lim _{h \rightarrow 0^{+}} P\left(\left\{N_{t_{i}}^{1}-N_{t_{i}-h}^{1}=1\right\}\right) P\left(\left\{N_{t_{i}}^{2}-N_{t_{i}-h}^{2}=1\right\}\right)=0 .
\end{aligned}
$$

We conclude that $P\left(\bigcup_{t \geq 0}\left\{\Delta N_{t}^{1}=1, \Delta N_{t}^{2}=1\right\}\right)=0$, which tell us that $N$ is a counting process. Furthermore, we have process defined by

$$
M_{t}:=N_{t}-\int_{0}^{t} \lambda_{s}^{1} \mathrm{~d} s-\int_{0}^{t} \lambda_{s}^{2} \mathrm{~d} s
$$

is a martingale. By the Watanabe's result, we have that $N$ is a Poisson process with intensity function $\lambda^{1}+\lambda^{2}$.

On the other hand, suppose that $N^{1}+N^{2}$ be a Poisson process, we aim to prove that $N^{1}$ and $N^{2}$ are independent. In fact, let $0 \leq t_{0} \leq t_{1} \leq \cdots \leq t_{n}$ and $u_{j} \in \mathbb{R}, j=0,1, \cdots, n-1$, we have

$$
\begin{aligned}
& \mathbb{E}\left(\exp \left\{i \sum_{j=0}^{n-1} u_{j}\left(N_{t_{j+1}}^{1}-N_{t_{j}}^{1}+N_{t_{j+1}}^{2}-N_{t_{j}}^{2}\right)\right\}\right) \\
& =\mathbb{E}\left(\mathbb{E}\left(\exp \left\{i \sum_{j=0}^{n-1} u_{j}\left(N_{t_{j+1}}^{1}-N_{t_{j}}^{1}+N_{t_{j+1}}^{2}-N_{t_{j}}^{2}\right)\right\} \mid \mathcal{F}_{t_{j}}\right)\right) \\
& =\mathbb{E}\left(\exp \left\{i \sum_{j=0}^{n-2} u_{j}\left(N_{t_{j+1}}^{1}-N_{t_{j}}^{1}+N_{t_{j+1}}^{2}-N_{t_{j}}^{2}\right)\right\} \mathbb{E}\left(\exp \left\{i u_{n}\left(N_{t_{n}}^{1}-N_{t_{n-1}}^{1}+N_{t_{n}}^{2}-N_{t_{n-1}}^{2}\right)\right\} \mid \mathcal{F}_{t_{n-1}}\right)\right) \\
& =\exp \left\{\mathrm{e}^{i u_{n}-1} \int_{t_{n-1}}^{t_{n}}\left(\lambda_{s}^{1}+\lambda_{s}^{2}\right) \mathrm{d} s\right\} \mathbb{E}\left(\exp \left\{i \sum_{j=0}^{n-2} u_{j}\left(N_{t_{j+1}}^{1}-N_{t_{i}}^{1}+N_{t_{j+1}}^{2}-N_{t_{j}}^{2}\right)\right\}\right) \\
& =\cdots=\exp \left\{\mathrm{e}^{\sum_{j=0}^{n-1}\left(u_{j}-1\right)}\left(\int_{t_{0}}^{t_{n}}\left(\lambda_{s}^{1}+\lambda_{s}^{2}\right) \mathrm{d} s\right)\right\} \\
& =\mathbb{E}\left(\exp \left\{i \sum_{j=0}^{n-1} u_{j}\left(N_{t_{j+1}}^{1}-N_{t_{j}}^{1}\right)\right\}\right) \mathbb{E}\left(\exp \left\{i \sum_{j=0}^{n-1} u_{j}\left(N_{t_{j+1}}^{2}-N_{t_{j}}^{2}\right)\right\}\right)
\end{aligned}
$$

which completes the proof.

We will recall the concept of conditional distribution from the measure theory. Let $\xi: \Omega \rightarrow E$ be a random element on $(\Omega, \mathcal{F}, P)$ taking value in a Polish space $(E, \mathcal{B}(E))$. Let $\mathcal{G} \subseteq \mathcal{F}$, then there exists a conditional distribution of $\xi$ with respect to $\mathcal{G}$, that is, a family $\left(Q_{\omega}\right)_{\omega \in \Omega}$ of probability measures on $(E, \mathcal{B}(E))$ such that

1) For any $A \in \mathcal{B}(E)$, the map $\omega \rightarrow Q_{\omega}(A)$ is $\mathcal{G}$-measurable;

2) For any $A \in \mathcal{B}(E), \quad D \in \mathcal{G}$,

$$
P(D \cap\{\xi \in A\})=\int_{D} Q_{\omega}(A) P(\mathrm{~d} \omega) .
$$

Remark 3.4. 1) The conditional distribution defined above is unique in the sense: if $\left(\tilde{Q}_{\omega}\right)_{\omega \in \Omega}$ is another family probability measures with the same properties, then $Q_{\omega}=\tilde{Q}_{\omega}$ for P-a.e. $\omega$.

2) If $A \in \mathcal{B}(E)$ is such that $P(\xi \in A)=1$, then $Q_{\omega}(A)=1$ for $P$-a.e. $\omega$.

Lemma 3.5. Let $(X, N)$ be a weak solution of (2.1) on a filtered probability space $\left(\Omega, \mathcal{F}, P,\left(\mathcal{F}_{t}\right)\right)$. Let $\left(Q_{\omega}\right)_{\omega \in \Omega}$ be a conditional distribution of $(X, N)$ with respect to $\mathcal{F}_{0}$ (here we consider $(X, N)$ as a $\mathbb{D} \times \mathbb{D}$ 
-valued random variable). We denote by $Y, M$ the canonical maps from $\mathbb{D} \times \mathbb{D}$ onto $\mathbb{D}$ respectively, that is

$$
Y: \mathbb{D} \times \mathbb{D} \rightarrow \mathbb{D}, \quad M: \mathbb{D} \times \mathbb{D} \rightarrow \mathbb{D},
$$

and

$$
Y\left(\omega_{1}, \omega_{2}\right):=\omega_{1}, \quad M\left(\omega_{1}, \omega_{2}\right):=\omega_{2}
$$

Let

$$
\begin{gathered}
\mathcal{H}_{t}=\mathcal{B}_{t}(\mathbb{D}) \otimes \mathcal{B}_{t}(\mathbb{D}), \\
\mathcal{H}=\vee_{t \geq 0} \mathcal{H}_{t} .
\end{gathered}
$$

Then, for $P$-a.e. $\omega$, the pair $(Y, M)$ is a weak solution of $(2.1)$ on $\left(\mathbb{D} \times \mathbb{D}, \mathcal{H}, Q_{\omega},\left(\mathcal{H}_{t}\right)\right)$.

Proof. Let us check the conditions of Definition 2.4.

1) Firstly, we will check that $M$ is an $\left(\mathcal{H}_{t}\right)$-Poisson process. For any $0 \leq s \leq t, \quad D \in \mathcal{H}_{s}, \quad A \in \mathcal{F}_{0}$, we have

$$
\begin{aligned}
& \mathbb{E}_{P}\left[\exp \left\{i u\left(N_{t}-N_{s}\right)\right\} I_{((X, N) \in D)} I_{A}\right] \\
& =\mathbb{E}_{P}\left[\exp \left\{i u\left(N_{t}-N_{s}\right)\right\}\right] \mathbb{E}_{P}\left[I_{((X, N) \in D)} I_{A}\right] \\
& =\exp \left\{\Lambda_{s, t}\left(\mathrm{e}^{i u-1}\right)\right\} \mathbb{E}_{P}\left[I_{((X, N) \in D)} I_{A}\right],
\end{aligned}
$$

where $\Lambda_{s, t}$ is defined as in Definition 2.1. Hence, we have

$$
\int_{A} E_{Q_{\omega}}\left[\exp \left\{i u\left(M_{t}-M_{s}\right)\right\} I_{D}\right] \mathrm{d} P=\int_{A} \exp \left\{\Lambda_{s, t}\left(\mathrm{e}^{i u-1}\right)\right\} Q_{\omega}(D) \mathrm{d} P .
$$

It follows that

$$
E_{Q_{\omega}}\left[\exp \left\{i u\left(M_{t}-M_{s}\right)\right\} I_{D}\right]=\exp \left\{\Lambda_{s, t}\left(\mathrm{e}^{i u-1}\right)\right\} Q_{\omega}(D) \quad P \text {-a.e. } \omega .
$$

Therefore, for $P$-a.e. $\omega$,

$$
E_{Q_{\omega}}\left[\exp \left\{i u\left(M_{t}-M_{s}\right)\right\} \mid \mathcal{H}_{s}\right]=\exp \left\{\Lambda_{s, t}\left(\mathrm{e}^{i u-1}\right)\right\} .
$$

We deduce that, for $P$-a.e $\omega, M$ is an $\left(\mathcal{H}_{t}, Q_{\omega}\right)$-Poisson process with intensity function $\lambda$.

2) For any $T \in[0, \infty)$,

$$
\int_{0}^{T}|b(t, X)| \mathrm{d} s+\int_{0}^{T}|g(s, X)| \mathrm{d} N_{s}<\infty \quad P \text {-a.e.. }
$$

By Remark 3.4, we have

$$
\int_{0}^{T}|b(t, Y)| \mathrm{d} s+\int_{0}^{T}|g(s, Y)| \mathrm{d} M_{s}<\infty \quad Q_{\omega} \text {-a.e., }
$$

for $P$-a.e. $\omega$.

3) We have

$$
X(t)=x+\int_{0}^{t} b(s, X) \mathrm{d} s+\int_{0}^{t} g(s, X) \mathrm{d} N_{s} \quad t \in[0, \infty), P \text {-a.e.. }
$$

Hence,

$$
Y(t)=x+\int_{0}^{t} b(s, Y) \mathrm{d} s+\int_{0}^{t} g(s, Y) \mathrm{d} M_{s} \quad t \in[0, \infty), Q_{\omega} \text {-a.e., }
$$

for $P$-a.e. $\omega$.

Proof of Theorem 3.1. Let $(X, N)$ be a weak solution of (2.1) on a filtered space $\left(\Omega, \mathcal{F}, P,\left(\mathcal{F}_{t}\right)\right)$. Let $N^{1}$ and $N^{2}$ be two independent $\left(\mathcal{F}_{t}^{\prime}, P^{\prime}\right)$-Poisson processes with the same intensity function $\lambda$. Set 


$$
\left(\tilde{\Omega}, \tilde{\mathcal{F}}, \tilde{P},\left(\tilde{\mathcal{F}}_{t}\right)\right):=\left(\Omega, \mathcal{F}, P,\left(\mathcal{F}_{t}\right)\right) \times\left(\Omega^{\prime}, \mathcal{F}^{\prime}, P^{\prime},\left(\mathcal{F}_{t}^{\prime}\right)\right) .
$$

Then $X, N, N^{1}$ and $N^{2}$ can be defined on $\tilde{\Omega}$ in an obvious way. The pair $(X, N)$ is a solution of (2.1) on $\left(\tilde{\Omega}, \tilde{\mathcal{F}}, \tilde{P},\left(\tilde{\mathcal{F}}_{t}\right)\right)$ and $N^{1}, N^{2}$ are independent $\left(\tilde{\mathcal{F}}_{t}, \tilde{P}\right)$-Poisson processes. For any $t \geq 0$, and $x \in \mathbb{D}$, $g(t, x)$ is a linear operator from $\mathbb{R} \rightarrow \mathbb{R}$. Let $\varphi(t, x)$ denote the orthogonal projection from $\mathbb{R} \rightarrow(\operatorname{ker} g(t, x))^{\perp}$; let $\psi(t, x)$ denote the orthogonal projection from $\mathbb{R} \rightarrow \operatorname{ker} g(t, x)$.

For any $0 \leq t$, set

$$
\begin{gathered}
\tilde{N}_{t}^{1}:=\int_{0}^{t} \varphi(s, X) \mathrm{d} N_{s}+\int_{0}^{t} \psi(s, X) \mathrm{d} N_{s}^{1}, \\
\tilde{N}_{t}^{2}:=\int_{0}^{t} \varphi(s, X) \mathrm{d} N_{s}^{2}+\int_{0}^{t} \psi(s, X) \mathrm{d} N_{s} .
\end{gathered}
$$

We claim that $\tilde{N}^{1}$ and $\tilde{N}^{2}$ are two independent $\left(\tilde{\mathcal{F}}_{t}, \tilde{P}\right)$-Poisson processes with the intensity function $\lambda$. In fact, it’s easy to see that $\tilde{N}^{1}, \tilde{N}^{2}$ and $\tilde{N}^{1}+\tilde{N}^{2}$ are counting processes. Moreover, Let

$$
\begin{gathered}
\tilde{M}_{t}^{1}:=\tilde{N}_{t}^{1}-\int_{0}^{t} \lambda_{s} \mathrm{~d} s, \\
\tilde{M}_{t}^{2}:=\tilde{N}_{t}^{2}-\int_{0}^{t} \lambda_{s} \mathrm{~d} s, \\
\tilde{M}_{t}^{3}:=\tilde{M}_{t}^{1}+\tilde{M}_{t}^{2} .
\end{gathered}
$$

We have

$$
\begin{aligned}
\tilde{M}_{t}^{1} & :=\int_{0}^{t} \varphi(s, X)\left(\mathrm{d} N_{s}-\lambda_{s} \mathrm{~d} s\right)+\int_{0}^{t} \psi(u, X)\left(\mathrm{d} N_{s}^{1}-\lambda_{s} \mathrm{~d} s\right), \\
\tilde{M}_{t}^{2} & :=\int_{0}^{t} \varphi(s, X)\left(\mathrm{d} N_{s}^{2}-\lambda_{s} \mathrm{~d} s\right)+\int_{0}^{t} \psi(u, X)\left(\mathrm{d} N_{s}-\lambda_{s} \mathrm{~d} s\right) .
\end{aligned}
$$

Note that processes $\varphi=\varphi(t, X)$ and $\psi=\psi(t, X)$ are predictable precesses and the integrators in the above equations are martingales. We conclude that $\tilde{M}^{1}$ and $\tilde{M}^{2}$ are martingale, theorefore $\tilde{M}^{3}$ is also a martingale. By Lemma 2.3, we get that $\tilde{N}^{1}$ and $\tilde{N}^{2}$ are two $\left(\tilde{\mathcal{F}}_{t}, \tilde{P}\right)$-Poisson processes with the intensity function $\lambda$ and $\tilde{N}^{1}+\tilde{N}^{2}$ is a $\left(\tilde{\mathcal{F}}_{t}, \tilde{P}\right)$-Poisson processes with the intensity function $2 \lambda$. By Lemma 3.3, we deduce that $\tilde{N}^{1}$ and $\tilde{N}^{2}$ are two independent $\left(\tilde{\mathcal{F}}_{t}, \tilde{P}\right)$-Poisson processes.

For any $t \geq 0$, we have

$$
\int_{0}^{t} g(s, X) \mathrm{d} N_{s}=\int_{0}^{t} g(s, X) \varphi(s, X) \mathrm{d} N_{s}=\int_{0}^{t} g(s, X) \mathrm{d} \tilde{N}_{s}^{1} .
$$

Consequently, $\left(X, \tilde{N}^{1}\right)$ is also a solution of (2.1) on $\left(\tilde{\Omega}, \tilde{\mathcal{F}}, \tilde{P},\left(\tilde{\mathcal{F}}_{t}\right)\right)$.

Let us now consider the filtration

$$
\mathcal{G}_{s}:=\tilde{\mathcal{F}}_{s} \vee \sigma\left(\tilde{N}_{t}^{2}: t \geq 0\right)=\tilde{\mathcal{F}}_{s} \vee \sigma\left(\tilde{N}_{t}^{2}-\tilde{N}_{s}^{2}: t \geq s\right),
$$

$s \geq 0$. Note that, for any $s \geq 0$, the $\sigma$-fields $\tilde{\mathcal{F}}_{s}$ and $\sigma\left(\tilde{N}_{t}^{1}-\tilde{N}_{s}^{1}: t \geq s\right) \vee \sigma\left(\tilde{N}_{t}^{2}-\tilde{N}_{s}^{2}: t \geq s\right)$ are independent. Thus, $\tilde{N}^{1}$ is a $\left(\mathcal{G}_{s}, \tilde{P}\right)$-Poisson process. So, the pair $\left(X, \tilde{N}^{1}\right)$ is also a solution of (2.1) on $\left(\tilde{\Omega}, \tilde{\mathcal{F}}, \tilde{P},\left(\mathcal{G}_{s}\right)\right)$.

Let $\left(Q_{\tilde{\omega}}\right)_{\tilde{\omega} \in \tilde{\Omega}}$ be a conditional distribution of $\left(X, \tilde{N}^{1}\right)$ with respect to $\mathcal{G}_{0}$. By Lemma 3.5, $(Y, M)$ is a solution of (2.1) on $\left(\mathbb{D} \times \mathbb{D}, \mathcal{H}, Q_{\tilde{\omega}},\left(\mathcal{H}_{t}\right)\right)$ for $\tilde{P}$-a.e. $\tilde{\omega}$. As the uniqueness in law holds for (2.1), the distribution $\operatorname{Law}\left(Y_{t} ; t \geq 0 \mid Q_{\tilde{\omega}}\right)$ (which is the conditional distribution of $X$ with respect to $\mathcal{G}_{0}$ ) is the same for 
$\tilde{P}$-a.e. $\tilde{\omega}$. This means that the process $X$ is independent of $\mathcal{G}_{0}$. In particular, $X$ and $\tilde{N}^{2}$ are independent.

For any $t \geq 0$ and $x \in \mathbb{D}$, let $g^{\dagger}(t, x)$ be the pseudo inverse of $g(t, x)$. It is easy to check that $g^{\dagger}=g^{\dagger}(t, x)$ is predictable and we have $g^{\dagger}(t, x) g(t, x)=\varphi(t, x)$. It follows that

$$
\int_{0}^{t} \varphi(s, X) \mathrm{d} N_{s}=\int_{0}^{t} g^{\dagger}(s, X) g(s, X) \mathrm{d} N_{s}=\int_{0}^{t} g^{\dagger}(s, X) \mathrm{d} N_{s}^{3},
$$

where

$$
N_{t}^{3}:=\int_{0}^{t} g(s, X) \mathrm{d} N_{s}=X_{t}-X-\int_{0}^{t} b(s, X) \mathrm{d} s .
$$

By (3.1), we get

$$
N_{t}=\int_{0}^{t} \varphi(s, X) \mathrm{d} N_{s}+\int_{0}^{t} \psi(s, X) \mathrm{d} N_{s}=\int_{0}^{t} g^{\dagger}(s, X) \mathrm{d} N_{s}^{3}+\int_{0}^{t} \psi(s, X) \mathrm{d} \tilde{N}_{s}^{2} .
$$

The process $N^{3}$ is a measurable functional of $X$, while $\tilde{N}^{2}$ is independent of $X$. Thus, the last equation shows that the distribution $\operatorname{Law}(X, N)$ is unique.

Remark 3.6. In this paper, the equivalence of the uniqueness in law and joint uniqueness in law holds when diffusion coefficient may be degenerate. We note that, for the general multidimensional stochastic differential equations with jumps, the equivalence does not hold when the diffusion coefficients are allowed to be degenerate. We will consider in the future study.

\section{Acknowledgements}

This work was supported in part by the National Natural Science Foundation of China(Grant No.11401029) and Zhejiang Provincial Natural Science Foundation of China (Grant No. LQ13A010020).

\section{References}

[1] Yamada, T. and Watanabe, S. (1971) On the Uniqueness of Solutions of Stochastic Differential Equations. Journal of Mathematics-Kyoto University, 11, 155-167.

[2] Ondrejat, M. (2004) Uniqueness for Stochastic Evolution Equations in Banach Spaces. Dissertationes Mathematicae, 426, 1-63.

[3] Bahlali, K., Mezerdi, B., N'zi, M. and Ouknine, Y. (2007) Weak Solutions and a Yamada Watanabe Theorem for FBSDEs. Random Operators and Stochastic Equations, 15, 271-286. http://dx.doi.org/10.1515/rose.2007.016

[4] Röckner, M.M., Schmuland, B. and Zhang, X. (2008) Yamada-Watanabe Theorem for Stochastic Evolution Equations in Infinite Dimensions. Condensed Matter Physics, 11, 247-259. http://dx.doi.org/10.5488/CMP.11.2.247

[5] Kurtz, T.G. (2007) The Yamada-Watanabe-Engelbert Theorem for General Stochastic Equations and Inequalities. Electronic Journal of probability, 12, 951-965. http://dx.doi.org/10.1214/EJP.v12-431

[6] Tappe, S. (2013) The Yamada-Watanabe Theorem for Mild Solutions to Stochastic Partial Differential Equations. Electronic Communications in Probability, 18, 1-13. http://dx.doi.org/10.1214/ECP.v18-2392

[7] Kurtz, T.G. (2013) Weak and Strong Solutions of General Stochastic Models. arXiv:1305.6747v1

[8] Graczyk, P. and Malecki, J. (2013) Multidimensional Yamada-Watanabe Theorem and Its Applications to Particle Systems. Journal of Mathematical Physics, 54, 021503. http://dx.doi.org/10.1063/1.4790507

[9] Barczy, M., Li, Z. and Pap, G. (2013) Yamada-Watanabe Results for Stochastic Differential Equations with Jumps. arXiv:1312.4485

[10] Zhao, H. (2014) Yamada-Watanabe Theorem for stochastic Evolution Equation Driven by Poisson Random Measure. ISRN Probability and Statistics, $7 \mathrm{p}$.

[11] Jean, J. (1980) Weak and Strong Solutions of Stochastic Differential Equations. Stochastics, 3, 171-191. http://dx.doi.org/10.1080/17442508008833143

[12] Engelbert, H. (1991) On the Theorem of T. Yamada and S. Watanabe, Stochastics. An International Journal of Probability and Stochastic Processes, 36, 205-216. http://dx.doi.org/10.1080/17442509108833718

[13] Brossard, J. (2003) Deux notions quivalentes dunicit en loi pour les quations diffrentielles stochastiques, Sminaire de Probabilits XXXVII. Springer Berlin Heidelberg, 246-250. 
[14] Cherny, A.S. (2002) On the Uniqueness in Law and the Pathwise Uniqueness for Stochastic Differential Equations. Theory of Probability and Its Applications, 46, 406-419. http://dx.doi.org/10.1137/S0040585X97979093

[15] Qiao, H.J. (2010) A Theorem Dual to Yamada-Watanabe Theorem for Stochastic Evolution Equations. Stochastics and Dynamics, 10, 367.

[16] Watanabe, S. (1964) On Discontinuous Additive Functionals and Lvy Measures of a Markov Process. Japanese Journal of Mathematics, 34, 53-70. 